\section{Should aPS/PT Be Incorporated into the Routine Serological Tests in the Diagnosis of Antiphospholipid Syndrome?}

\section{To the Editor:}

We read with great interest the article by Zohoury, et al ${ }^{1}$ on how to close the serological gap in the diagnosis of antiphospholipid syndrome (APS) by using non-criteria antiphospholipid antibodies (aPL). In their well-designed study, the authors found that using 4 of 11 non-criteria tests [antiphosphatidylserine/prothrombin complex (aPS/PT), antiphosphatidylserine (aPS), antiphosphatidylethanolamine antibodies, and anticardiolipin $(\mathrm{aCL}) /$ vimentin antibodies], an accumulative $30.9 \%$ of seronegative APS (SN-APS) patients were detected, and there was a further $5.9 \%$ increase when using the other 7 non-criteria tests. On the basis of their findings, the authors concluded that patients displaying clinical features of APS but negative for conventional criteria markers should undergo additional testing for non-criteria biomarkers.

Among those non-criteria biomarkers, aPS/PT has exhibited the most promising potential owing to the availability of the well-characterized and standardized commercial ELISA kits ${ }^{2}$. In this letter, we hope to contribute to this discussion by calling attention to an additional report that we recently published on the clinical relevance of aPS/PT in Chinese patients with APS $^{3}$.

In our study, sera from 441 subjects were analyzed, including sera from 101 patients with primary APS (PAPS), 140 patients with secondary APS (SAPS), 34 patients with non-APS thrombosis, 49 patients with non-APS pregnancy-related morbidity, 78 patients with systemic lupus erythematosus, and 39 healthy controls. Among the 241 patients with APS, 31 patients were SN-APS, as suggested by other studies ${ }^{4}$. Those patients fulfilled the clinical criteria for APS, but were negative for the 3 traditional aPL [lupus anticoagulant (LAC), IgG/IgM aCL, and $\mathrm{IgG} / \mathrm{IgM}$ anti- $\beta_{2}$-glycoprotein I (anti- $\left.\left.\beta_{2}-\mathrm{GPI}\right)\right]^{5}$. Study protocols were reviewed and approved by the Ethics Committee of Peking Union Medical College Hospital and informed consent was obtained from all participants. Serum aCL (IgG and $\operatorname{IgM})$, anti- $\beta_{2}$-GPI (IgG and $\operatorname{IgM})$, and aPS/PT (IgG and $\operatorname{IgM}$ ) were determined by ELISA (QUANTA Lite ELISA, Inova). We found that $\operatorname{IgG}$ and IgM aPS/PT were present in $29.7 \%$ and $54.5 \%$ of PAPS, and $42.1 \%$ and $53.6 \%$ of SAPS, respectively. In addition, IgG aPS/PT correlated with venous thrombosis.
Importantly, IgM/IgG aPS/PT were detected in $22.6 \%$ of SN-APS $(13.3 \%$ in SN-PAPS and $31.3 \%$ in SN-SAPS), a proportion higher than the results of Zohoury, et $a l^{1}$. Interestingly, 2 recent studies from China ${ }^{6,7}$ also showed the presence of $\operatorname{IgM} / \mathrm{IgG}$ aPS/PT in SN-APS. One study reported that $\mathrm{IgM} / \mathrm{IgG}$ aPS/PT were detected in $20 \%$ of SN-APS ${ }^{6}$, while the other study indicated that $\mathrm{IgM} / \mathrm{IgG}$ aPS/PT were present in $51 \%$ of SN-APS ${ }^{7}$. In addition to the results from China, data from Europe ${ }^{1,4}$ also reported the existence of $\mathrm{IgM} / \mathrm{IgG}$ aPS/PT in SN-APS, ranging from $9.4 \%(12 / 128)^{4}$ to $11.8 \%(8 / 68)^{1}$, further highlighting that $\mathrm{IgM} / \mathrm{IgG}$ aPS/PT may enhance the diagnostic performance of traditional aPL panel for APS.

As a supplement to our previous study ${ }^{3}$, we further assessed the added value provided by $\operatorname{IgM} / \mathrm{IgG}$ aPS/PT regarding the APS standard laboratory diagnostic panel ${ }^{5}$. As shown in Table 1, we found that the combination of $\mathrm{IgM} / \mathrm{IgG}$ aPS/PT with LAC displayed a sensitivity of $52.70 \%$, a specificity of $99.40 \%$, and an LR+ value of 84.84 in the diagnosis of APS, higher than those from the combinations of $\mathrm{IgM} / \mathrm{IgG}$ aCL with $\mathrm{LAC}$ and $\mathrm{IgM} / \mathrm{IgG}$ anti- $\beta_{2}$-GPI with LAC. Importantly, the combination of $\operatorname{IgM} / \mathrm{IgG}$ aPS/PT with IgM/IgG aCL and LAC resulted in a sensitivity of $38.60 \%$ and a specificity of $100 \%$. In addition, the combination of IgG aPS/PT with IgG anti- $\beta_{2}$-GPI and LAC exhibited a better correlation with arterial thrombosis than other aPL combinations (Figure 1).

We agree with the conclusions presented by Zohoury, et al ${ }^{1}$ that the non-criteria biomarkers should be tested in patients displaying clinical features of APS but negative for conventional criteria markers, a practice also suggested by the 2010 International APS Congress ${ }^{8}$. However, the question we emphasize in this letter is whether we should consider incorporating aPS/PT into the routine serological tests in the diagnosis of APS. First, when the 2006 international consensus statement ${ }^{5}$ was proposed, the detection of aPS/PT was mainly based on in-house ELISA, resulting in large variability among different studies. Over the past 10 years, the performance of ELISA-based systems for detection of aPS/PT has substantially improved, and commercially available assays with improved sensitivity and specificity have been evaluated in many studies. Second, aPS/PT covers a significant proportion of SN-APS, and the combination of aPS/PT with traditional aPL further enhances the diagnostic power. Third, the introduction of aPS/PT further strengthens risk stratification in patients with APS. Our updated knowledge in aPL and the development of new assays keep moving the field

Table 1. The predictive power of combination of various aPL in the diagnosis of APS. Except for likelihood ratios (LR), data are percentages.

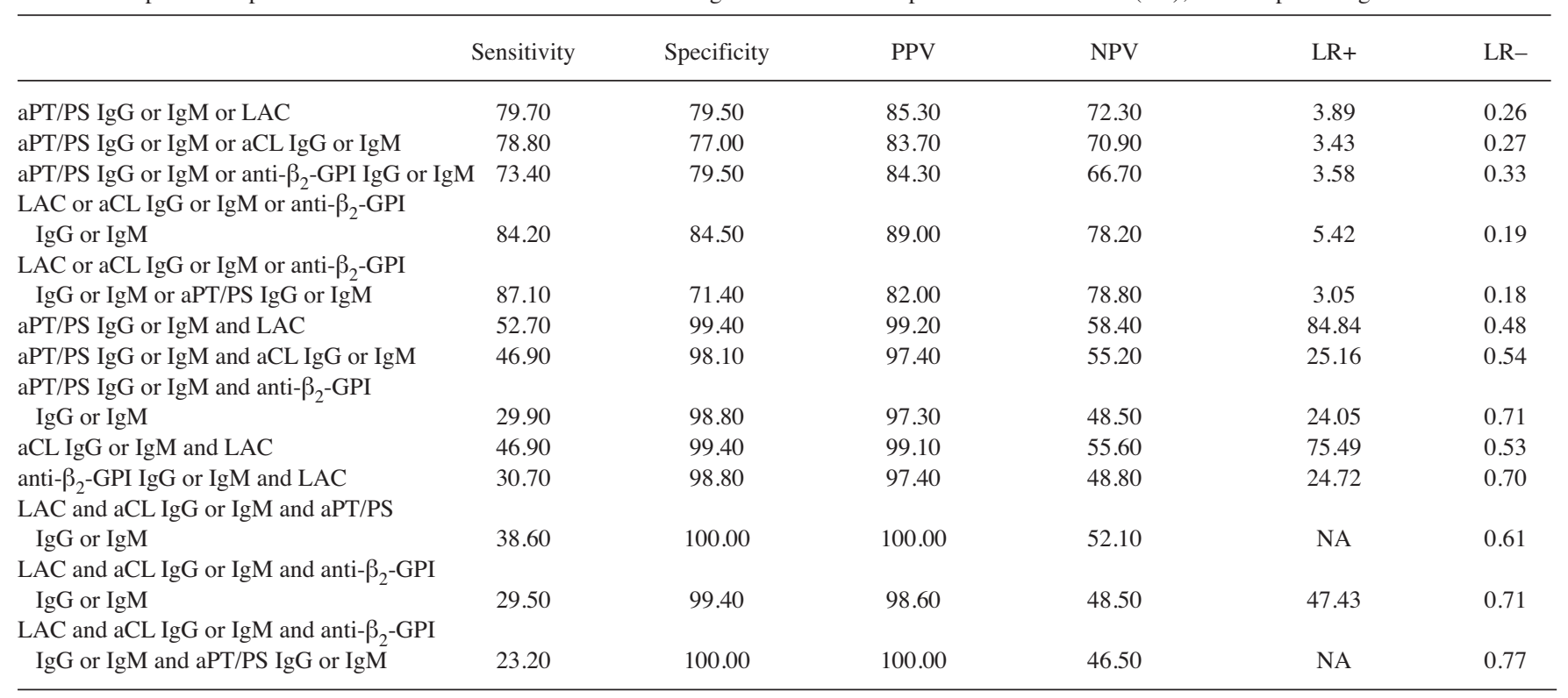

aPL: antiphospholipid antibodies; APS: antiphospholipid syndrome; PPV: positive predictive value; NPV: negative predictive value; NA: not applicable; aPT/PS: antiphosphatidylserine/prothrombin antibodies; aCL: anticardiolipin antibodies; anti- $\beta_{2}$-GPI: anti- $\beta_{2}$-glycoprotein I; LAC: lupus anticoagulants.

Personal non-commercial use only. The Journal of Rheumatology Copyright @ 2019 . All rights reserved. 

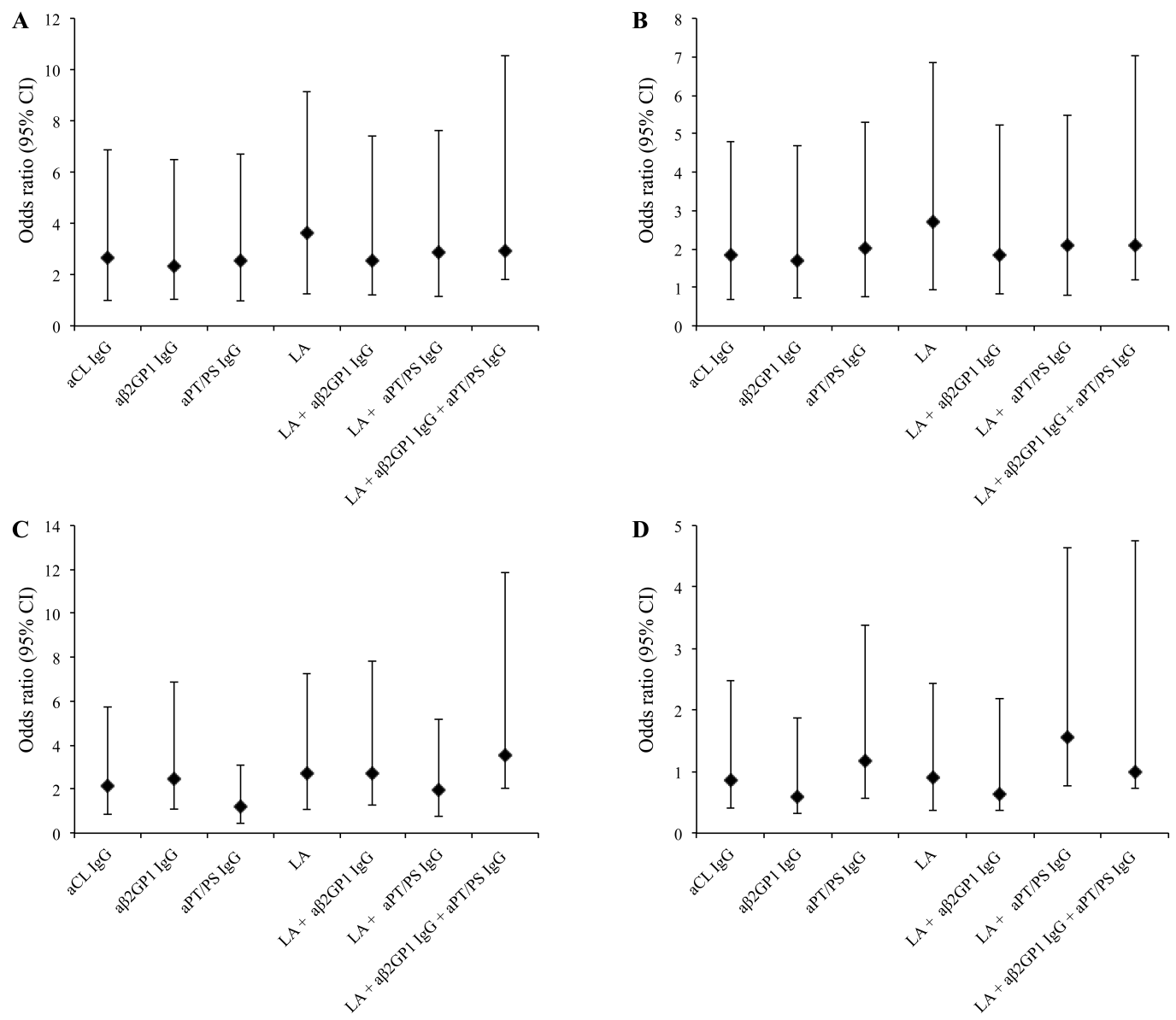

Figure 1. Correlations between multiple aPL and thrombosis (A), venous thrombosis (B), arterial thrombosis (C), and obstetrical complications (D). aPL: antiphospholipid antibodies; aPT/PS: antiprothrombin/antiphosphatidylserine antibodies; aCL: anticardiolipin antibodies; a $\beta 2 \mathrm{GPI}$ : anti- $\beta_{2}$-glycoprotein I; LA: lupus anticoagulants.

forward for both patients and clinicians in the clinical and therapeutic decision-making process.

SHULAN ZHANG, MD; FENGCHUN ZHANG, MD; YONGZHE LI, MD; Department of Rheumatology and Clinical Immunology, Peking Union Medical College Hospital, Chinese Academy of Medical Sciences and Peking Union Medical College, Key Laboratory of Rheumatology and Clinical Immunology, Ministry of Education, Beijing, China. This work was supported in part by the National Natural Science Foundation of China Grants No. 81373188, 81671618 (to YL), 81771661 (to SZ), Chinese Academy of Medical Sciences (CAMS) Initiative for Innovative Medicine (CAMS-I2M) No. 2017-I2M-3-001 (to SZ and YL), The National Key Research and Development Program of China No. 2016YFC0903900 (to YL). Address correspondence to Dr. Y. Li, Department of Rheumatology and Clinical Immunology, Peking Union Medical College Hospital, Chinese Academy of Medical Sciences and Peking Union Medical College, Key Laboratory of Rheumatology and Clinical Immunology, Ministry of Education, No. 1 Shuai Fu Yuan, Eastern District, Beijing 100730, China. E-mail: LiYZ@ pumch.cn or yongzhelipumch@126.com

\section{REFERENCES}

1. Zohoury N, Bertolaccini ML, Rodriguez-Garcia JL, Shums Z, Ateka-Barrutia O, Sorice M, et al. Closing the serological gap in the antiphospholipid syndrome: The value of "non-criteria" antiphospholipid antibodies. J Rheumatol 2017;44:1597-602.

2. Amengual O, Forastiero R, Sugiura-Ogasawara M, Otomo K, Oku $\mathrm{K}$, Favas C, et al. Evaluation of phosphatidylserine-dependent antiprothrombin antibody testing for the diagnosis of antiphospholipid syndrome: results of an international multicentre study. Lupus 2017;26:266-76.

3. Zhang S, Wu Z, Zhang W, Zhao J, Norman GL, Zeng X, et al. Antibodies to phosphatidylserine/prothrombin (aPS/PT) enhanced the diagnostic performance in Chinese patients with antiphospholipid syndrome. Clin Chem Lab Med 2018;56:939-46.

4. Hoxha A, Ruffatti A, Mattia E, Meneghel L, Tonello M, Salvan E, et al. Relationship between antiphosphatidylserine/prothrombin and conventional antiphospholipid antibodies in primary antiphospholipid syndrome. Clin Chem Lab Med 2015;53:1265-70.

5. Miyakis S, Lockshin MD, Atsumi T, Branch DW, Brey RL, Cervera $\mathrm{R}$, et al. International consensus statement on an update of the classification criteria for definite antiphospholipid syndrome (aps). J Thromb Haemost 2006;4:295-306.

6. Zhu L, Li C, Liu N, Yang X, Jia RL, Mu R, et al. Diagnostic value of antibodies to phosphatidylserine/prothrombin complex for antiphospholipid syndrome in Chinese patients. Clin Rheumatol 2017;36:401-6.

7. Shi H, Zheng H, Yin YF, Hu QY, Teng JL, Sun Y, et al. Antiphosphatidylserine/prothrombin antibodies (aPS/PT) as potential diagnostic markers and risk predictors of venous 
thrombosis and obstetric complications in antiphospholipid

syndrome. Clin Chem Lab Med 2018;56:614-24.

8. Bertolaccini ML, Amengual O, Atsumi T, Binder WL, de Laat B,

Forastiero R, et al. 'Non-criteria' aPL tests: report of a task force and preconference workshop at the 13th International Congress on

Antiphospholipid Antibodies, Galveston, TX, USA, April 2010.

Lupus 2011;20:191-205.

J Rheumatol 2019;46:1; doi:10.3899/jrheum.171402 